\title{
Phase-field Modeling of Brittle Fracture and its Adaptive Moving Mesh Solution
}

\author{
Weizhang Huang*, Xianping $\mathrm{Li}^{\dagger}$, Fei Zhang ${ }^{\S}$ and Shicheng Zhang ${ }^{\S}$ \\ * Department of Mathematics, University of Kansas, Lawrence, Kansas 66049, USA \\ e-mail:whuang@ku.edu, web page: https://whuang.ku.edu \\ ${ }^{\dagger}$ College of Integrative Sciences and Arts, Arizona State University, Mesa, Arizona 85212, USA \\ ${ }^{\S}$ College of Petroleum Engineering, China University of Petroleum, Changping, Beijing 102249, \\ China
}

\begin{abstract}
The phase-fieldmodeling and its adaptive moving mesh solution for studying initiation and propagation of brittle fracture will be presented. Challenges such as non-smoothness of the energy functional, violation of fracture boundary conditions, and the need for mesh adaptation, and possible remedies for these challenges will be discussed. In particular, a moving mesh finite element method will be presented for the numerical solution of the phase-field model for brittle fracture.
\end{abstract}

\section{REFERENCES}

[1] F. Zhang, W. Huang, X. Li, and S. Zhang, "Moving mesh finite element simulation for phasefield modeling of brittle fracture and convergence of Newton's iteration", J. Comput. Phys., Vol. 356, pp. 127-149, (2018).

[2] F. Zhang, W. Huang, X. Li, and S. Zhang, "A study on phase-field models for brittle fracture", arXiv 1805.07357. 